\title{
Periodic Lensing from a Photoemitted Electron Gas Undergoing Cyclotron Oscillations
}

\author{
Omid Zandi, Allan Sykes, Ryan Cornelius, Francis Alcorn, and Renske van der Veen
}

University of Illinois at Urbana-Champaign, Urbana, Illinois, United States

Dynamics of a photo-emitted electron gas in a uniform magnetic field in real time is observed by Ultrafast Transmission Electron Microscopy (UTEM) [1]. In UTEM, ultrafast electron pulses are used to probe the sample while ultrafast laser pulses, synchronized to the electron pulses, initiate dynamics in the sample [2]. Our UTEM, depicted in Fig. 1(a), is based on a custom-modified Hitachi 9500 environmental TEM, where an added section below the acceleration stage guides an ultraviolet pulsed laser beam to a $\mathrm{LaB}_{6}$ carbon guard-ring photocathode. The electron pulses are accelerated and guided onto the sample to produce either images, diffraction patterns or spectra, while the sample is being irradiated by tunable laser pulses through the EDS port. An active feedback system with four cameras stabilizes the beam pointing on the cathode and sample. The delay between the laser pump and electron probe pulses is controlled using an optical delay line such that light-induced dynamics can be captured with a temporal resolution better than 1 ps.

In this work, a $\sim 150 \mathrm{fs}, 520 \mathrm{~nm}$ laser pulse photo-creates an electron gas in a two-photon photoemission process from a copper grid (Fig. 1b). The electron gas undergoes a collective cyclotron oscillation under the influence of its own Coulomb field as well as the external, uniform magnetic field from the objective lens in the TEM. At the laser incidence time, i.e. time zero, the photoelectrons gain an initial dispersive kinetic energy due to their Coulomb interactions. Consequently, the magnetic field confines the electron gas in the transverse plane (perpendicular to the magnetic field) and periodically brings the electrons back to their initial positions as much as the Coulomb force allows.

The electron gas undergoes radial breathing oscillations with a frequency that is slightly lower than what would be expected given the static magnetic field at the sample position. This reduction in frequency is assigned to Coulomb interactions between the electrons. The cloud expands freely along the magnetic field direction and the space charge effect becomes weaker over time. When the electron gas radially shrinks to its minimum size and adopts a prolate shape, it behaves like a diverging lens to the probing electrons in the UTEM, magnifying the image of the copper grid, as shown in Fig. 2(a). Integrating the signal intensity at each time step in a region of interest (ROI) where the magnification is observed, results in the time trace shown in Fig. 2(b). It shows that the signal is periodic and exceptionally long-lived, and it decays exponentially due to dispersion and absorption of the electrons.

We developed an analytical model for the focal length $f(t)$ of electron gas under the assumption that space charge effects are negligible:

$f(t)=-2(2 \pi)^{3 / 2} \varepsilon_{0} \gamma v_{z}^{2} m \sigma_{r}^{2} /\left(N e^{2}\right) \times\left[2\left(\sigma_{v} /\left(\omega \sigma_{r}\right)\right)^{2}(1-\cos (\omega t))+1\right] e^{t / \tau}$

where $N, m, e, \sigma_{v}, \sigma_{r}$ and $\varepsilon_{0}$ are the number of electrons in cloud, their mass, charge, transverse velocity spread, initial transverse spatial standard deviation, and permittivity of free space, respectively, and $v_{z}$ and $\gamma$ are the velocity of the probe electrons and their Lorentz gamma factor, respectively. The cyclotron angular frequency is $\omega=e B_{0} / m$, where $B_{0}$ is the magnetic field of the UTEM objective lens. The damping of the lensing effect is modeled by the exponential term $\mathrm{e}^{\mathrm{t} / \tau}$, where the damping time constant $\tau$ is determined by fitting the lens equation to the experimental ROI data. In this way, we derived an electron- 
gas focal length between $\sim-4$ and $-0.5 \mathrm{~m}$ over the period of one cyclotron oscillation. This is an order-ofmagnitude estimate that depends on the number of electrons in the cloud.

In addition, we performed N-body simulations to verify the model and compare to the experiment. Of interest is the effect of the grounded copper grid on the behavior of the electron gas. The lensing effect is weaker during the very first few ps after laser excitation (second frame in Fig. 2a) compared to the subsequent reconvention of the electrons due to the magnetic field (fourth frame in Fig. 2a). This is because of the positive image charges in the copper grid and the oblate geometry of the cloud at early time delays. Our study shows that with a laser fluence of $33 \mathrm{~mJ} / \mathrm{cm}^{2}$ and pulse duration of fs exciting a 3000 copper mesh grid sample with $34 \%$ open area and with no extraction field, the number of photoemitted electrons is in the order of 100,000 with a velocity spread in the order of $4 \times 10^{5} \mathrm{~m} / \mathrm{s}$. The results from this study pave the way for a better understanding of photoemission processes, electron Coulomb explosions in a uniform magnetic field, and the collective cyclotron dynamics of charge carries in free space as well as inside materials.

(a)

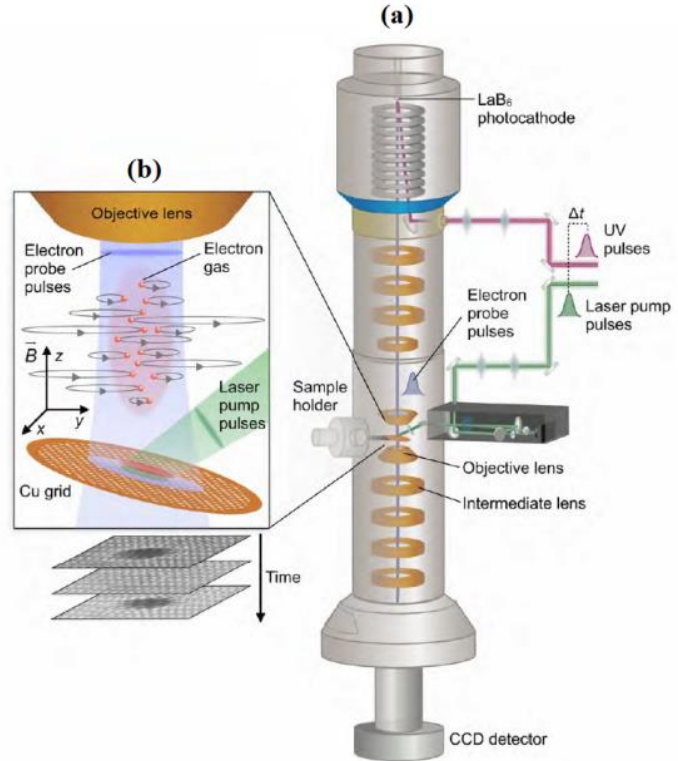

Figure 1. (a) Schematic of the UTEM setup; (b) A photoemitted electron gas inside the uniform magnetic field of the objective lens shows collective cyclotron oscillations that cause periodic lensing in the probe electrons leading to a magnification of the grid image.
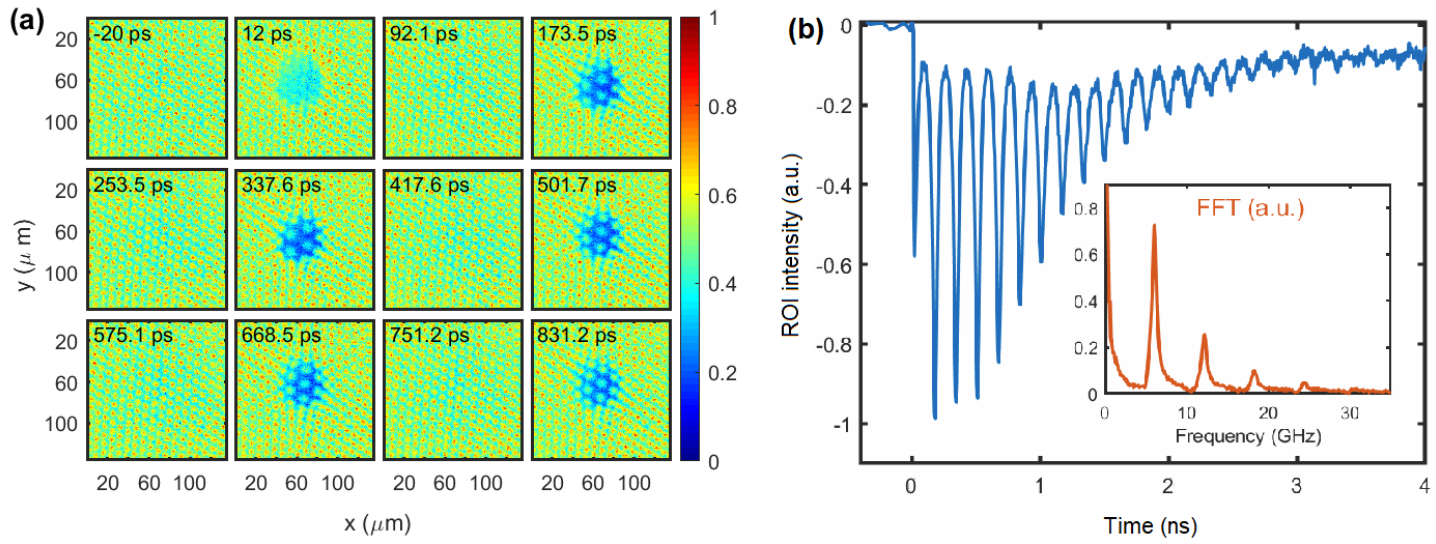
Figure 2. (a) Periodic lensing causes a magnification in the image of a copper grid recorded by UTEM. The laser excites the copper at $t=0$. (b) Region-of-interest (ROI) trace as a function of time and its fast Fourier transform (FFT) in the inset. The ROI is depicted by a red circle in the last image of part (a)

References

[1] Zandi, Omid, Allan E. Sykes, Ryan D. Cornelius, Frank M. Alcorn, Brandon Zerbe, Phillip M. Duxbury, Bryan W. Reed, and Renske M. van der Veen. "Transient lensing from a photoemitted electron gas imaged by ultrafast electron microscopy." arXiv preprint arXiv:2001.01389 (2020).

[2] Zewail, Ahmed H. "Four-dimensional electron microscopy." Science 328, no. 5975 (2010): 187-193. 